\title{
Toward positive psychosocial practice in psychosis: In pursuit of subjective wellbeing in severe mental ill- health
}

\author{
Pawel D. Mankiewicz
}

\begin{abstract}
This article summarises and reflects on the scarce literature on the subject of positive psychosocial practice in the clinical specialism of complex and enduring mental health needs, such as psychosis. An attempt is made to demonstrate that such practice is not only achievable among individuals with severe psychological difficulties but, indeed, has already begun to develop, although it seems still in its infancy. The literature reviewed in this paper appears to indicate that a person with psychosis is as capable of experiencing subjective wellbeing as any other person in the general population. However, in order to promote wellbeing and sustained recovery among such individuals, a specialist psychosocial input needs to be delivered in a positive - that is integrative, person-based, collaborative, socially inclusive, and flow-inducing - manner. Furthermore, the article endeavours to demonstrate that in order to effect a fundamental shift in the perception of severe mental ill-health from a deficit-based and psychopathology-oriented stance toward a person-based and socially inclusive one, the principles of positive practice need to inform a wide range of clinical and social activities, including assessment, intervention, interpersonal reengagement and public policy development. It is nevertheless acknowledged that positive psychosocial approaches to psychosis are still in their infancy and relevant research studies remain considerably underrepresented, and in many aspects virtually non-existent. Hence, it is suggested that future research in positive clinical psychology within the specialty of complex, severe and enduring mental ill-health is actively encouraged and pursued by both clinicians and academics.
\end{abstract}

Keywords: subjective wellbeing, positive psychosocial practice, positive clinical psychology, psychosis, severe mental ill-health

\section{Introduction}

A rather restricted focus on pathology has for a long time dominated clinical applications of the psychological discipline and has resulted in a relatively negativistic model of human experiences. As argued by Seligman and Csikszentmihalyi (2000), such a model appears deficient of the positive features that make life worth living and distorts fundamental understanding of normal emotional functioning. Particularly in clinical psychology, the neglect of subjective wellbeing (SWB) might be explained by the field's long-established focus on the more severe, complex and enduring forms of psychological difficulties, which in practice often means addressing problems and reducing distress rather than promoting happiness (Conway \& MacLeod, 2002). Although examples of studies describing successfully-implemented interventions and their positive impact on the wellbeing of people with psychosis have been available in the clinical literature, Braehler and Harper (2008) still reported that the quality of life of those diagnosed with schizophrenia 
remained low. Yet, more than two decades ago, Romme and Escher (1993) proposed that rehabilitation of people with psychosis should focus on enhancing all fundamental qualities of their lives. Similarly, Chadwick (1997) demonstrated that such individuals have virtues as well as deficits, and psychological interventions needed to focus on their strengths in order to increase the dignity and SWB of those with psychosis.

Using semantic and bibliographic methods, Rusk and Waters (2013) analysed over 1.7 million documents in 700 journals covering a range of psychosocial disciplines and concluded that approximately 4 percent of the published papers belonged to applied positive psychology. Regrettably, to date such studies among individuals with severe mental health needs appear considerably under-represented in comparison with psychopathologically focused clinical research. Given the historic endorsement of the disease paradigm of emotional difficulties and neglect of individual virtues in clinical psychology, it appears important for clinicians working within the specialism of severe mental ill-health to recognise how aspects of positive psychology, such as SWB models, apply to their clients, and how such models may inform the development of positive clinical practice aimed at supporting and increasing their clients' life satisfaction. In order to arrive at such inferences, it needs to be shown that despite the generally upheld negativistic portrayals of psychosis, it is possible to reliably measure SWB levels within this clinical population. It also needs to be demonstrated that individuals with complex psychological problems are able to experience happiness, and that employment of SWB models is attainable with such persons within both therapeutic practice and broader socially inclusive interventions.

This article attempts to review and summarise relevant literature on the subject of utilisation of positive psychology principles among one of the most socially disadvantaged and stigmatised clinical populations, that is, those with active experiences of psychosis, and to propose how such principles may shift the functional goal of psychosocial interventions from a temporary reduction of distress to ensuring a prolonged enhancement of one's SWB as the means for a longterm recovery. This article investigates how the innovative movement of positive clinical psychology may contribute to fundamental change in the way clinicians think about severe mental health needs, from a deficit-based and psychopathologically oriented stance towards a person-based and socially inclusive one. Based on the reviewed literature, the article argues that individuals with enduring psychoses are as capable of experiencing SWB as those from the general population, and illustrates how positive psychosocial interventions may enhance such experiences within the discussed clinical group.

Furthermore, an attempt is made to argue that positive psychosocial practice in psychosis is principally about a particular (i.e. integrative, person-based, collaborative, inclusive, and flowinducing) way that more traditional and empirically established therapeutic interventions, such as those based on cognitive-behavioural models, are delivered, and about the explicitly defined values governing such interventions. As argued by Linley, Joseph, Harrington and Wood (2006), in order to develop and establish a positive therapeutic practice, a full integration of traditional evidence-based and deficit-oriented models of therapy with positive psychology principles needs to be promoted. Here, it is proposed that such an integrative approach is not only achievable among clients with psychosis but, indeed, it has already begun to develop.

\section{Refuting the disease paradigm of schizophrenia}

It seems of principal importance for mental health professionals to revisit the terminology they use to describe experiences of psychosis. Harper (2001) proposed that particular descriptions of psychological problems influence the range of interventions offered to the clients. As argued by Maddux (2002), due to the long established reliance on psychiatric concepts, clinical psychology 
practice may have also become pathologised, and the assumptions of the disease paradigm continue as implicit guides to psychological treatments, which aim to alleviate disordered conditions that reside within the person and are thought of as symptoms of illness. Yet, research studies appear to demonstrate that bio-genetic explanations of psychosis increase fear and prejudice towards diagnosed individuals, and lead to their social marginalisation (Geekie, 2004). Furthermore, Campbell (2007) illustrated how those diagnosed with schizophrenia felt trapped within the stigmatising and discriminatory professional framework, which in their opinion encouraged the dismissal of significant aspects of their experience.

Although the medical construct of schizophrenia has been methodologically refuted for decades (Bentall, 1990), psychosis continues to be typically described using a diagnostic approach. Consequently, Mankiewicz (2013) argued that in order to counterbalance such an unhelpful clinical tradition, the growing importance of psychosocial concepts of psychosis should encourage clinicians to offer a choice of individually tailored explanations and interventions, and support individuals in making sense of their experiences in the context of their lives. Such practice focusing on self-defining abilities of a client would potentially have an empowering, healing effect.

\subsection{Pessimistic comorbidity}

Severe emotional distress has been acknowledged in most aetiological models of psychosis, whilst high levels of depression and anxiety were found to be prevalent amongst people with diagnoses of schizophrenia (Marneros \& Akiskal, 2007). The prevalence of diagnostic comorbidity was found to be as high as $57.3 \%$, of which approximately $62 \%$ of people were found to have anxiety disorder (Good, 2002). Siris (1991) reviewed 29 studies investigating the incidence of secondary depression in psychosis, and concluded that its occurrence ranged to as many as $70 \%$ of studied cases.

Overall, emotional wellbeing in psychosis as portrayed in the majority of clinical and research literature appears to be largely pessimistic. Such bias, as noticed by Social Exclusion Unit (2004) and Royal College of Psychiatrists (2009), might have contributed to a discouraging supposition that the levels of SWB among individuals with psychosis were considerably lower than those of the general population, and in consequence, those individuals would be unable to become satisfied with their lives. Hence, in order to examine the validity of such supposition, the positive psychological practice in psychosis ought to incorporate a comprehensive assessment of wellbeing levels among individuals with such experiences.

\section{Toward positive assessment in psychosis}

Huppert and So (2013) emphasised the significance of the measurement of SWB as a recovery indicator in mental health. As stated by the authors, high SWB equates with positive mental health and functionally may be perceived as lying at the opposite end of a spectrum to common psychological problems. Such a perspective might be illustrated by the four-dimension model of SWB and psychological distress developed by Headey and Wearing (1992). The researchers provided evidence that some psychological distress can occur alongside moderately high general levels of happiness, yet both were negatively correlated. Thus, it was proposed that a comprehensive assessment of one's psychological wellbeing needed to incorporate both positive and negative aspects of one's experience. Subsequently, Headey and Wearing (1992) demonstrated that depression and anxiety, the two widespread forms of psychological distress, were moderately correlated and both had effects on happiness through their inverse influence 
on life satisfaction (cognitive dimension of SWB) and positive emotion (affective component of SWB).

Yet, it appears the assessment of SWB serves as an indicator of more than one's general mental health. Forgeard, Jayawickreme, Kern \& Seligman (2011) argued that such assessments would indicate the quality of individual experiences within the wider social context. The authors emphasised that "subjective measures appear to be indispensible insofar as the presence of objective conditions of wellbeing may not always be accompanied with subjective feelings of wellbeing" (p. 98).

\subsection{Assessment of SWB in psychosis}

In order to address the apparent absence of research literature addressing SWB assessment among people with psychosis, Mankiewicz, Gresswell and Turner (2013a) conducted a clinical study in which they examined the utilisation of SWB measures among individuals with active experiences of hallucinations and paranoia. Two standardised instruments, widely used in positive psychology research, were employed. The Satisfaction with Life Scale (Diener, Emmons, Larsen \& Griffin, 1985) was used to investigate the levels of cognitive dimension of SWB, while the Positive Affect Scale, a standardised subtest of the Affect Balance Scale (Bradburn, 1969), was utilised to examine the affective component of participants' happiness. The authors demonstrated that SWB measures could be reliably administered to individuals with active psychoses, including those on acute mental health wards (Mankiewicz et al., 2013a). Interestingly, not only was Headey and Wearing's model with its correlational values successfully replicated, but the sample's mean for positive affect approximated the levels established in the general population, indicating that the participants exhibited average levels of positive affect. Thus, contrary to the negative inferences derived from the disease paradigm of schizophrenia, the participants exhibited levels of positive affect typical for the universal average. Descriptive data indicated that the measurement scores approximated normal distribution.

Subsequently, in order to utilise the integrative principle of positive psychological practice postulated by Joseph and Linley (2006) within the process of assessment of individuals with psychosis, Mankiewicz, Gresswell and Turner (2013b) investigated levels of psychological distress and examined how experiences of psychosis affected the satisfaction with life of such individuals. Indeed, the presence of psychosis was accompanied by increased levels of psychological distress, and the sample's life satisfaction mean was placed within the 'slightly dissatisfied' score range. The scores were still normally distributed. Furthermore, the study outcomes indicated that the effects that experiences of psychosis, such as paranoid beliefs, had on individual life satisfaction were mediated by the levels of emotional distress experienced by individuals. A mediational analysis of data demonstrated that depression acted as a dominant mediator in the association between the experiences of psychosis and satisfaction with life.

\subsection{Happiness in psychosis}

Based on the outcomes of their research, Mankiewicz et al. (2013b) proposed a number of conclusions to inform positive psychological practice in psychosis. Their conclusions are summarised in Table 1 below. 
Table 1. Null effects of psychosis on SWB levels - summary of clinical study conclusions (Mankiewicz et al., 2013b).

\begin{tabular}{l}
\hline Null effect \\
\hline $\begin{array}{l}\text { Psychosis does } \\
\text { not equal } \\
\text { unhappiness }\end{array}$ \\
Psychosis does \\
not exempt \\
individuals from \\
positive mood set- \\
points
\end{tabular}

Summary of clinical study conclusions

The levels of SWB among people diagnosed with paranoid schizophrenia were largely similar to the levels typical for the general population. The range of sample scores demonstrated that individuals experiencing psychosis could feel very joyful and be satisfied with their lives. Hence, contrary to the deficit-oriented portrayal of psychosis, empirical data suggested that individuals with such experiences are able to report levels of SWB similar to those in the general population.

There seems to be a positive, rather than neutral, affect baseline in humans, which has significant adaptive, evolutionary, motivational, social, learning and intrinsic functions (Diener \& Diener, 1996). The exact set-point varies among individuals, depending on a person's socialisation and temperament, yet for most people it remains in the positive range. Since the general levels of positive affect in psychosis were demonstrated to be similar to those reported in the general population, the experiences of psychosis did not appear to automatically perturb positive mood set-points among the study participants.

Psychosis does The results suggested that experiences of psychosis would indirectly not indiscriminately reduce life satisfaction affect individual life satisfaction through their influence on depression levels. If auditory hallucinations and/or delusional beliefs become activated (despite use of medication) and are appraised by an individual as a loss of control over their lives, the feelings of helplessness and hopelessness are elicited. In depression, self-defeating cognitions seem unconditional and over-generalised, hence negatively affect individual appraisal of one's entire life (Gilbert, 2009).

Psychosis does The positive affect levels among individuals with psychosis were not immobilize adaptive mechanisms of SWB similar to those reported in the general population, hence appeared intact. Due to the stable levels of positive affect, despite clearly elevated experiences of psychological distress, the average satisfaction with life was only slightly lowered in comparison with general population trends. The dynamic equilibrium theory of happiness (Headey, 2006) suggests that major life events are typically habituated to and people soon return to their usual, individually-set equilibrium states of SWB, despite initially increased levels of psychological distress and reduced levels of positive affect. Empirical data produced by Mankiewicz et al. (2013b) indicated that individuals diagnosed with paranoid schizophrenia were able to habituate to such disconcerting experiences as onset of psychosis, acute episodes of paranoia and hearing voices, or admissions to inpatient mental health wards, and with time returned to their individual SWB equilibrium states. 


\section{Toward positive psychological interventions in psychosis}

Given the results of the above study, Mankiewicz et al. (2013b) suggested that in order to enhance life satisfaction among individuals with psychosis, psychological interventions would need to focus initially on reducing experiences of depression. And traditional clinical psychology emphasising the alleviation of emotional distress offers exactly such interventions. However, applied positive psychology provides an alternative approach that complements the traditional deficit-based stance. In other words, positive therapists may offer a range of strength-based interventions that promote individual SWB and buffer against mental ill-health (Joseph \& Linley, 2006). The aforementioned four-dimension model of SWB and psychological distress seems to depict accurately how the enhanced SWB levels may operate as individual shields against psychological distress. Such a functional shift in clinical settings may be achieved only through a full integration of the positive and negative aspects of human experience, so that psychological therapists can empower their clients in finding individually tailored ways to alleviate distress and promote wellbeing and optimal functioning (Linley et al., 2006).

\subsection{Toward positive cognitive-behavioural therapy}

Several analyses of current evidence in the specialism of psychological interventions for psychosis demonstrated the effectiveness of cognitive-behavioural therapy for psychosis (CBTp). In their meta-analytical evaluation of controlled research and qualitative reviews, Roth and Fonagy (2005) presented numerous studies with favourable outcomes. Also, CBTp has been supported with affirmative results of case studies (e.g. Mankiewicz \& Turner, 2014) and randomised control trials (e.g. Farhall, Freeman, Shawyer \& Trauer, 2009; Jolley et al., 2003).

As argued by Mankiewicz (2013), the novel cognitive-behavioural advances in working with psychosis could potentially help mental health professionals to shift their perception of such experiences from a pathological syndrome to a non-pathological difficulty that may cause considerable distress and decrease SWB. Yet, can a range of cognitive-behavioural interventions be employed within a positive therapeutic framework? And, if so, what criteria would need to be met to insure that the delivered behaviourally-based therapy is indeed positive in its nature?

Those enquiries were explored by Weiss and Knoster (2008), who argued that such therapy needed to move away from the traditional mechanistic utilisation of its interventions and become person-based. Subsequently, the authors proposed that in order for a therapeutic model to meet criteria of positive psychological intervention it must allow the client opportunity to express opinions and exert a level of control over their life through meaningful choices, help the person to identify their fundamental needs and positively influence the client's quality of life. Furthermore, the authors argued that positive behavioural approaches ought to be overtly based on therapeutic collaboration (versus authoritarian control) and functionally focused on illumination, i.e. understanding the meanings and purposes of the individual's behaviour from their subjective perspective, rather than exclusively on elimination, i.e. extinguishing unhelpful behaviours.

Even earlier, Keyes and Lopez (2002) proposed that positive treatments were those that had an objective to build upon a person's existing strengths and eliminate barriers to personal growth. Once more, the major importance of integration of the positive (promoting wellbeing) and negative (addressing signs of psychological distress) was highlighted. The authors emphasised that such therapies do not differ from traditional models in their content, but rather in their explicit focus. Likewise, research studies reviewed by Thompson (2002) demonstrated how a range of conventional cognitive-behavioural interventions (such as goal setting, cognitive restructuring, stress reduction and problem-solving skills, positive mental imagery, and self- 
instruction training) were effectively employed to increase the individuals' subjective quality of life.

Subsequently, Fava and Ruini (2003) proposed that wellbeing-oriented psychological interventions should be integrated with traditional approaches and delivered after a range of problematic cognitions and behaviours have been addressed. Also, Frish (2006) demonstrated that traditional cognitive-behavioural therapy could be successfully combined with positive psychology principles, and utilised across a wide spectrum of mental health needs. Both models explicitly address unhelpful cognitions, difficult emotions and dysfunctional behaviours through numerous cognitive and behavioural therapeutic strategies, yet complement the traditional CBT approach with an integrated wellbeing component.

An intervention based on such integrative principles was investigated by Seligman, Rashid and Parks (2006), who studied the effectiveness of positive psychotherapeutic input in alleviating depression. The CBT-derived treatment integrated interventions focusing on negative and positive experiences and incorporated both cognitive strategies and behavioural tasks. The authors demonstrated that the treatment led to a considerable symptomatic relief, even among severely depressed clients. The effect sizes were moderate to large. Similarly, the effectiveness of cognitive therapy based on the notions of positive psychology was investigated by Padash, Dehnavi and Botlani (2012). The delivered intervention considerably increased individuals' SWB with particular gains in life satisfaction, despite various psychological problems, such as depression.

\subsection{Toward positive CBTp}

Considering the discussed principles of positive cognitive-behavioural therapies, it appears that CBTp may potentially be delivered within the positive framework. Indeed, as argued below, it seems that such adaptations of CBTp have already begun to be developed and utilised.

Lopez and Kerr (2006) emphasised that positive therapy practice needs to be based on core values of shared therapeutic goals, collaborative work, and reciprocally attainable results. Likewise, the crucial importance of adopting a person-centred stance in positive therapies was emphasised by Joseph and Linley (2006). As argued by the authors, in positive psychological practice, despite an initial focus on alleviating distressing symptoms, the clients ought to be supported in finding outlets and expression for their directional tendencies that are congruent with their intrinsic values. Such positive shift in the therapeutic focus would provide an empowering alternative to the disease model of mental illness, and thus appears particularly important for those with severe, complex and enduring psychological needs.

In 1996, Chadwick, Birchwood and Trower predicted that although CBTp had initially helped clinicians to move away from the medical constructs of schizophrenia and liberated psychological research and practice, it would one day need to step beyond its symptom focus and turn towards an ordinary human psychology, that is psychology of the person. Subsequently, Chadwick (2006) proposed a fundamental shift in cognitive-behavioural practice for psychosis and endorsed Person-Based CBTp, in which interventions were based on a collaborative therapeutic relationship and employed as a conceptual rather than manualised process, and thus empowered clients in achieving their personal goals. The empowering therapeutic alliance in person-based CBTp was argued by Mankiewicz (2013) to be central in establishing rapport with often socially stigmatised and excluded individuals.

Yet ultimately, as proposed by King and Ollendick (2008), the decisive criterion in measuring the individual suitability of a particular therapeutic model is its acceptability to the clients. Such a position undermines the old psychiatric orthodoxy and explicitly challenges the dominance 
and power of an expert in the process of psychological intervention. Indeed, in the study conducted by Farhall et al. (2009) the general levels of clients' acceptability of CBTp and their satisfaction ratings were shown to be high, and the mean client-rated working alliance appeared excellent.

\section{Toward social re-engagement in psychosis}

An ongoing debate about the relevance of external factors in SWB continues to take place in positive psychology. It seems that, unless the basic life needs are jeopardized, most people tend to report positive levels of their life satisfaction (Diener \& Diener, 1996). That is, most individuals in the general population tend to be 'rather satisfied' with their lives, unless exposed to adverse circumstances. Such a conclusion appears particularly relevant to those with severe psychological difficulties, since, as demonstrated by Mankiewicz et al. (2013 b), subjective emotional distress, with depression as a dominant mediator, accounted for $22.4 \%$ of variance in life satisfaction among those with active experiences of psychosis.

Indeed, more than two decades ago, Pilgrim (1990) argued that the most important dimensions of lives of people with psychosis were underscrutinised. The overpowering dominance of the disease paradigm of schizophrenia might have distorted the fact that most of those with enduring psychoses remained poor, unemployed, homeless or lived in deprived circumstances and often experienced exploitation and multiple traumas (Read, Seymour \& Mosher, 2004).

\subsection{Combating social exclusion}

As argued by Lingwood (2006), people with severe mental ill-health have long been located at the edges of community life and were among the most excluded of all social groups, experiencing widespread stigma and discrimination. The Royal College of Psychiatrists (RCP, 2009) defined social exclusion as "the extent to which individuals are unable to participate in key areas of economic, social and cultural life" (p. 1). Non-participation arising from constraints rather than choice was emphasised. Interestingly, among the main reasons for widespread social exclusion of those with complex psychological needs, as identified by the Social Exclusion Unit (SEU, 2004), were low expectations and negative assumptions held by mental health professionals about the capabilities of individuals with psychiatric diagnoses. Indeed, the SEU reported that service users frequently raised concerns about how clinicians' negative attitudes toward their abilities would often delay their recovery.

Bergsma and Veenhoven (2011) investigated the levels of SWB and functionality of social participation among individuals with a range of mental health needs. The researchers demonstrated that those with psychiatric diagnoses who experienced higher levels of SWB were more socially engaged, less absent from work, and required less multidisciplinary support. The authors concluded that individuals with psychological difficulties felt happier if they presented with social characteristics of those with good mental health. They would still experience symptoms, yet were able to cope and consequently feel more satisfied with their lives. Hence, the importance of improving social conditions for people with mental health needs was emphasised. The authors argued that "the better external living conditions in society, the more the remaining difference in happiness depends on inner life ability" (p. 3). Therefore it seems imperative that the variance in SWB affected by societal circumstances is acknowledged and addressed through a range of supportive psychosocial interventions.

The significance of measuring and enhancing SWB of people with psychosis in order to counteract exclusion, stigma and discrimination has been voiced by service users for years 
(Campbell, 2007). As a result, Chadwick (2006) emphasised that clinicians pursuing personbased CBTp in their practice must attempt to conceptualise individual experiences of psychosis in the context of a person's whole life. In practice, it seems therefore essential to incorporate the client-related factors that are socially bound and may influence the individual's therapeutic goals.

\subsection{Toward social recovery in psychosis}

As argued by SEU (2004), mental health services need to empower individuals with complex psychological needs to regain the things they value in their lives regardless of their diagnoses. This requires a more positive approach from the services in order to recognise and accommodate individual needs. Such a positive approach would need to incorporate, firstly, breaking the assumed link between mental ill health and social incompetence, and assisting individuals in making personal choices about their lives, and secondly, encouraging broader social participation, including occupational and leisure activities, to promote life satisfaction.

Interestingly, Tew et al. (2012) argued that clinical recovery within mental health was not essential for an individual to achieve considerable social progress, and emphasised that individuals with psychological difficulties were largely able to recover socially despite the continuous presence of clinical symptoms. Based on a comprehensive literature review, the authors concluded that rebuilding a worthwhile life, irrespective of whether or not individuals continued to experience particular psychological difficulties, was most central in reclaiming their valued social roles, positive self-identities and life satisfaction.

Even earlier, James (2001) suggested that overcoming a sense of powerlessness might be one of the key factors increasing SWB among people with psychosis. However, Tew et al. (2012) employed a more holistic stance toward recovery and argued that it needed to involve a journey of both personal change and social reengagement. The authors identified a number of processual and contextual social recovery factors that would increase wellbeing among individuals with mental health needs. These included transformation from an illness-dominated identity to an agency and competency-based one, participation in the community, identification of individual strengths and resources within a wider environment, development of social connectedness based on a subjective sense of belonging, and engagement in subjectively rewarding activities.

\subsection{Toward optimal experiences in psychosis}

It appears plausible to suggest that most conclusions derived from the literature on the subject of recovery in severe psychological difficulties appear consistent with the key notions of the theory of flow developed by Csikszentmihalyi (2002). The theory proposed that SWB can be enhanced through engagement with everyday life and involvement in absorbing tasks, such as work or leisure activities, in which individuals exercise their strengths, talents and interests. The intrinsic absorption in activities that people do for their own sake would eventually lead to the unique harmony and order in consciousness, i.e. flow. Such optimal experiences help individuals cultivate an optimistic perception of their lives and thus prevent them from experiencing psychological ill-health. These suppositions informed the practical recommendations that Carr (2005) gave to mental health professionals aspiring to pursue a positive psychology approach in their clinical practice. For instance, the author suggested that clinicians might help clients identify their abilities and talents, and explore ways to use these frequently to generate optimal experiences.

Given the crucial role that activities and hobbies play in building psychological health among people with severe mental health problems (Bryson, Lysaker \& Bell, 2002), a clinical case example 
of a successful delivery of flow interventions to a client with enduring psychosis reported two decades ago by Csikszentmihalyi (1997) should not come as a surprise. Csikszentmihalyi's experience sampling method was used with a female client after more than a decade of her continuous inpatient hospitalisation to identify a range of flow-inducing activities. Subsequently, such activities (e.g. taking care of her fingernails) were supported with further input encouraging the development of personal competencies (e.g. participation in a professional manicurist course) to ensure the frequency of optimal experiences. Following a considerable enhancement in SWB, despite the preceding unresponsiveness to medical and pharmacological treatments, the person was discharged to community and within a year became self-sufficient.

Furthermore, utilisation of CBTp interventions may also play an important role in encouraging social reengagement. For instance, French, Morrison, Walford, Knight, and Bentall (2003) presented a case example of a successfully implemented CBTp with an individual experiencing delusional beliefs. The intervention not only ameliorated the person's emotional distress but also enabled the individual's social functioning through engaging in intrinsically rewarding interpersonal activities.

In the UK, numerous clinical guidelines for socially inclusive practice have emerged. For instance, the SEU (2004) argued that individuals with mental ill-health have much to offer socially, and if they were supported in fulfilling their potentials, the constraining impact of their psychological difficulties would be considerably reduced. Hence, the SEU recommended that clinicians promote the social inclusion of their clients through offering support in finding ways to accomplish their aspirations, access opportunities, and participate in a range of leisure activities. Additionally, the National Social Inclusion Programme (NSIP, 2009) emphasised that people with complex mental ill-health ought to be supported in developing their skills and talents, so that they become able to live fulfilling lives in a way that promotes and sustains their SWB.

A number of community-based initiatives involving 'social prescribing' by clinicians were developed by the SEU (2004). Prescriptions for learning, exercise and arts were utilised as key interventions. Furthermore, positive approaches to the education of mental health practitioners have been employed through provision of training in combating discrimination toward individuals with psychosis and in eliciting their qualities and strengths (Houghton, Shaw, Hayward \& West, 2006).

\section{Toward positive mental health policies}

As argued by Weiss and Knoster (2008), positive behaviourally-oriented psychological input needs to be embedded in the wider culture of social inclusion and empowerment. The authors insisted that all mental health practitioners have an important responsibility to the people they serve "that has less to do with getting others to act in predetermined ways and much more to do with supporting people to become increasingly self-determined and fulfilled" (p. 77). The pertinent role of social recovery, that is building one's life beyond the constraints of a psychological problem without necessarily achieving full clinical recovery, was emphasised in the UK in RCP's Position Statement (2009) as a way to promote social inclusion for individuals with mental health needs. Regaining a sense of personal control and participating in developmental opportunities were emphasised among the key factors allowing social recovery to occur. Social inclusion focused not only on overcoming objective barriers that people with psychosis would come up against, but also on helping individuals to address their subjective desire for a fulfilling role in their social lives. 
Forgeard et al. (2011) recognised the quality of social support in combination with sense of accomplishment and competence as central indicators of individual wellbeing, which, as argued by the authors, ought to be explicitly supported through relevant developments in public policies. Since employment rates among people with severe and enduring mental health needs were estimated as $3.4 \%$ (Dominy \& Hayward-Butcher, 2012), occupational reengagement in psychosis appears central to one's recovery. Indeed, a study conducted by Dominy and Hayward-Butcher (2012) demonstrated significant gains in subjective quality of life among people with such difficulties following employment.

In recent years, a gradual shift toward the development of mental health policies that are informed by the principles of positive clinical practice might have been observed in the UK. Utilisation of such policies within the specialism of complex mental ill-health has the apparent potential to generate a radical change in how individuals with psychoses are supported in their communities. For instance, in Capabilities for Inclusive Practice, the Department of Health (2007) recommended that mental health professionals conduct their assessments in a way that informs a holistic understanding of individuals, including their strengths and capacities. Consequently, psychological care was to be provided in the context of the specific aspirations of a person, in order to promote their social reengagement. Similarly, the NSIP (2009) emphasised that one of the main functions of a socially reengaging practice in complex mental health was to ensure a meaningful and intrinsically motivating participation in community life.

As a result of such policies, an increasing number of specialist community mental health services for individuals with psychosis appear to have adopted the socially inclusive recommendations. Such services follow the process of deinstitutionalisation, employ assertive outreach support, have a shared goal of facilitating independence, enable functional interpersonal networks, and improve social interactions. For instance, Davies, Hopkins, Campisi and Maggs (2012) illustrated a successful development and provision of a high relational supportive service for individuals with severe and enduring psychological difficulties, including psychosis. The authors reported significant gains in the areas of social integration, independence and exercised choice among the service users. Additionally, despite the continuous presence of symptoms (such as delusional beliefs), an improvement in individual wellbeing was also recorded.

\subsection{Accessibility of psychological therapies for psychosis}

In 2007, New Ways of Working for Applied Psychologists, a joint initiative between the British Psychological Society and the National Institute for Mental Health in England, emphasised the centrality of psychological formulation in planning and delivering mental health services. A formulation approach was openly contrasted with the illness model of emotional distress, which represented the pervasive discourse in services. The recent advances in understanding of psychosis and growing evidence for effectiveness of psychological therapies were argued as imperative in providing an open challenge to the dominant medical constructs of psychological difficulties.

The critical role of equal access to psychological therapies across the entire spectrum of severity of mental health needs was emphasised by the SEU (2004). Consequently, in 2009, the National Institute for Health and Care Excellence (NICE) in the UK published its clinical guideline for psychosis, in which CBTp and family-focused interventions were recommended as core psychological treatments for all individuals with such needs. The guideline was recently reviewed and the recommendations for psychological treatments were upheld (NICE, 2014). 
Nonetheless, despite the promising developments in mental health policies and psychosocial practice affecting individuals with psychoses, a range of organisational barriers to the delivery of effective services was still identified in a clinical study conducted by Mankiewicz and Turner (2012). The recognised obstacles included, interestingly, clinicians' pessimism related to the effectiveness of the interventions offered. The authors recommended that such reservations are openly acknowledged and addressed through regular consultation and psychoeducation targeting the therapists' pessimism and creating a more hopeful, encouraging and, indeed, positive attitude toward the offered services and their recipients.

\section{Conclusions and future directions}

In this article an attempt was made to demonstrate that a positive clinical psychology practice is not only achievable among individuals with the most complex and severe mental health needs, such as psychosis, but indeed has already begun to develop, although it seems still to be in its infancy. The scarce literature on the subject of positive psychosocial practice in psychosis reviewed in this paper appears to indicate that in order to effect a fundamental shift in clinicians' perception of such difficulties from a deficit-based and psychopathology-oriented stance toward a person-based and socially inclusive one, and to promote a sustained recovery among affected individuals, the principles of positive practice in psychosis need to inform a wide range of clinical and social activities, including assessment, intervention, interpersonal reengagement, and public policy development.

An attempt was made to demonstrate that people with psychoses are as capable of experiencing affirmative levels of SWB as any other person in the general population. Yet, in order to promote wellbeing among such individuals, a specialist psychological input needs to be delivered in an integrative, person-based, collaborative, socially inclusive, and flow-inducing manner. It was proposed that evidence-based cognitive-behavioural therapies may in some instances be delivered as positive interventions to those with complex, severe and enduring mental health needs. To achieve this, the positive psychology principles ought to be functionally incorporated, so that they explicitly guide the delivery of such interventions and empower the clients in the pursuit of fulfilling lives. Person-based CBTp was used as an innovative example of a therapeutic modality for psychosis-type difficulties that approximates a clinically applied positive psychology framework.

However, it needs to be acknowledged that positive psychosocial approaches to psychosis are still in their infancy. Clinically relevant research studies remain considerably underrepresented in the discussed specialism and, in many aspects, appear virtually nonexistent. Thus, it seems essential that future research in positive clinical psychology within the specialty of complex, severe and enduring mental ill-health is actively encouraged and pursued by both clinicians and academics. Such research would need to adopt a holistic approach to individual wellbeing in psychosis, and examine both internal (psychological) and external (objective) indicators of one's SWB.

\section{Authors}

Pawel D. Mankiewicz

National Health Service, UK

pawel.mankiewicz@nhs.net

\section{Publishing Timeline}

Received 2 July 2015 
Accepted 3 November 2015

Published 18 December 2015

\section{References}

Bentall, R. P. (1990). Reconstructing schizophrenia. London, UK: Routledge.

Bergsma, A., \& Veenhoven, R. (2011). The happiness of people with a mental disorder in modern society. Psychology of Well-Being: Theory, Research and Practice, 1(2), 1-6. http://dx.doi.org/10.1186/2211-1522-1-2

Bradburn, N. M. (1969). The structure of psychological well-being. Chicago, IL: Aldine Publishing Company.

Braehler, C., \& Harper, S. F. (2008). Identifying psychological needs in psychosis - perceptions and priorities of CMHT keyworkers. Clinical Psychology Forum, 182, 13-17.

British Psychological Society (2007). New ways of working for applied psychologists in health and social care: Organising, managing, and leading psychological services. Leicester, UK: Author.

Bryson, G., Lysaker, P., \& Bell, M. (2002). Quality of life benefits of paid work activity in schizophrenia. Schizophrenia Bulletin, 28(2), 249-257. http://dx.doi.org/10.1093/oxfordjournals.schbul.a006935

Campbell, P. (2007). Hearing my voice. The Psychologist, 20(5), 298-299.

Carr, A. (2005). Positive psychology. Clinical Psychology, 45, 5-6.

Chadwick, P. (2006). Person-based cognitive therapy for distressing psychosis. Chichester, UK: Wiley. http://dx.doi.org/10.1002/9780470713075

Chadwick, P., Birchwood, M., \& Trower, P. (1996). Cognitive therapy for delusions, voices and paranoia. Chichester, UK: John Wiley \& Sons.

Chadwick, P. (1997). Schizophrenia: The positive perspective. New York, NY: Brunner-Routledge.

Conway, C., \& MacLeod, A. (2002). Well-being: Its importance in clinical practice and research. Clinical Psychology, 16, 26-29.

Csikszentmihalyi, M. (1997). Finding Flow: The psychology of engagement with everyday life. New York, NY: Perseus Books Group.

Csikszentmihalyi, M. (2002). Flow: The classic work on how to achieve happiness. London, UK: Rider.

Davies, J., Hopkins, M., Campisi, M., \& Maggs, R. G. (2012). Developing high relational support services for individuals with long term mental health needs: Scheme description and service evaluation. Mental Health and Social Inclusion, 16(1), 31-40. http://dx.doi.org/10.1108/20428301211205883

Department of Health (2007). Capabilities for inclusive practice. London, UK: Author.

Diener, E., \& Diener, C. (1996). Most people are happy. Psychological Science, 7(3), 181-185. http://dx.doi.org/10.1111/j.1467-9280.1996.tb00354.x

Diener, E., Emmons, R. A., Larsen, R. J., \& Griffin, S. (1985). The Satisfaction with Life Scale. Journal of Personality Assessment, 49(1), 71-75. http://dx.doi.org/10.1207/s15327752jpa4901 13

Dominy, M., \& Hayward-Butcher, T. (2012). “Is work good for you?” Does paid employment produce positive social capital returns for people with severe and enduring mental health conditions? Mental Health and Social Inclusion, 16(1), 14-25. http://dx.doi.org/10.1108/20428301211205865

Farhall, J., Freeman, N. C., Shawyer, F., \& Trauer, T. (2009). An effectiveness trial of cognitive-behaviour therapy in a representative sample of outpatients with psychosis. British Journal of Clinical Psychology, 48(1), 47-62. http://dx.doi.org/10.1111/j.2044-8260.2009.tb00456.x

Fava, G. A., \& Ruini, C. (2003). Development and characteristics of a well-being psychotherapeutic strategy: Well-being therapy. Journal of Behavior Therapy and Experimental Psychiatry, 34(1), 45-63. http://dx.doi.org/10.1016/S0005-7916(03)00019-3

Forgeard, M. J. C., Jayawickreme, E., Kern, M. L., \& Seligman, M. E. P. (2011). Doing the right thing: Measuring wellbeing for public policy. International Journal of Wellbeing, 1(1), 79-106.

French, P., Morrison, A. P., Walford, L., Knight, A., \& Bentall, R. P. (2003). Cognitive therapy for preventing transition to psychosis in high-risk individuals: A case series. Behavioural and Cognitive Psychotherapy, 31, 53-67. http://dx.doi.org/10.1017/s1352465803001061

Frish, M. B. (2006). Quality of life therapy: Applying a life satisfaction approach to positive psychology and cognitive therapy. Hoboken, NJ: John Wiley \& Sons. 
Geekie, J. (2004). Listening to the voices we hear: Clients' understandings of psychotic experiences. In J. Read, L. R. Mosher, \& R. P. Bentall (Eds.), Models of madness: Psychological, social and biological approaches to schizophrenia (pp. 147-160). Hove, UK: Brunner-Routledge.

Gilbert, P. (2009). Overcoming depression: A self-help guide using cognitive-behavioural techniques (3rd ed.). London, UK: Robinson.

Good, J. (2002). The effect of treatment of a comorbid anxiety disorder on psychotic symptoms in a patient with a diagnosis of schizophrenia: A case study. Behavioural and Cognitive Psychotherapy, 30, 347-350. http://dx.doi.org/10.1017/s1352465802003090

Harper, D. (2001). Psychiatric and psychological concepts in understanding psychotic experience. Clinical Psychology, 7, 21-27.

Headey, B. (2006). Happiness: Revisiting set-point theory and dynamic equilibrium theory to account for long term change. Discussion Papers, 607, 1-18.

Headey, B., \& Wearing, A. (1992). Understanding happiness: A theory of subjective well-being. Melbourne, Australia: Longman Cheshire.

Houghton, P., Shaw, B., Hayward, M., \& West, S. (2006). Psychosis revisited: Taking a collaborative look at psychosis. Mental Health Practice, 9(6), 40-3. http://dx.doi.org/10.7748/mhp2006.03.9.6.40.c1905

Huppert, F. A., \& So, T. T. C. (2013). Flourishing across Europe: Application of a new conceptual framework for defining well-being. Social Indicators Research, 110(3), 837-861.

http://dx.doi.org/10.1007/s11205-011-9966-7

James, A. (2001). Raising our voices: An account of the Hearing Voices movement. Gloucester, UK: Handsell Publishing.

Jolley, S., Garety, P., Craig, T., Dunn, G., White, J., \& Aitken, M. (2003). Cognitive therapy in early psychosis: A pilot randomised controlled trial. Behavioural and Cognitive Psychotherapy, 31, 473-478. http://dx.doi.org/10.1017/S1352465803004107

Joseph, S., \& Linley, P. A. (2006). Positive therapy. A meta-theory for positive psychological practice. London, UK: Routledge.

Keyes, C. L. M., \& Lopez, S. J. (2002). Toward a science of mental health: Positive directions in diagnosis and interventions. In C. R. Snyder \& S. J. Lopez (Eds.), Handbook of positive psychology (pp. 45-59). New York, NY: Oxford University Press.

King, N. J., \& Ollendick T. H. (2008). The elegant psychosocial intervention: A heuristic conceptual framework for clinicians and researchers. Behavioural and Cognitive Psychotherapy, 36, 253-261. http://dx.doi.org/10.1017/S1352465808004359

Lingwood, L. (2006). Building bridges to social inclusion. In C. Jackson \& K. Hill (Eds.), Mental health today: A handbook (pp. 21-27). Brighton, UK: Pavilion Publishing.

Linley, P. A., Joseph, S., Harrington, S., \& Wood, A. M. (2006). Positive psychology: past, present, and (possible) future. Journal of Positive Psychology, 1(1), 3-16.

http://dx.doi.org/10.1080/17439760500372796

Lopez, S. J., \& Kerr, B. A. (2006). An open souce approach to creating positive psychological practice: A comment on Wong's strengths-centred therapy. Psychotherapy: Theory, Research, Practice, Training, 43(2), 147-150. http://dx.doi.org/10.1037/0033-3204.43.2.147

Maddux, J. E. (2002). Stopping the madness: Positive psychology and the deconstruction of the illness ideology and the DSM. In C.R. Snyder \& S.J. Lopez (Eds.), Handbook of positive psychology (pp. 13-25). New York, NY: Oxford University Press.

Mankiewicz, P. D. (2013). Cognitive-behavioural symptom-oriented understanding of psychosis: Abandoning the disease paradigm of schizophrenia. Counselling Psychology Review, 28(1), 53-63.

Mankiewicz, P. D., Gresswell, D. M., \& Turner, C. (2013a). Happiness in severe mental illness: Exploring subjective wellbeing of individuals with psychosis and encouraging socially inclusive multidisciplinary practice. Mental Health and Social Inclusion, 17(1), 27-34. http://dx.doi.org/10.1108/20428301311305287

Mankiewicz, P. D., Gresswell, D. M., \& Turner, C. (2013b). Subjective wellbeing in psychosis: Mediating effects of psychological distress on happiness levels amongst individuals diagnosed with paranoid schizophrenia. International Journal of Wellbeing, 3(1), 35-59. http://dx.doi.org/10.5502/ijw.v3i1.3 
Mankiewicz, P. D., \& Turner, C. (2012). Do Assertive Outreach clients with experiences of psychosis receive the NICE recommended cognitive-behavioural interventions? An audit. Clinical Psychology Forum, 240, 32-37.

Mankiewicz, P. D., \& Turner, C. (2014). Cognitive restructuring and graded behavioural exposure for delusional appraisals of auditory hallucinations and comorbid anxiety in paranoid schizophrenia. Case Reports in Psychiatry, 14, 1-8. http://dx.doi.org/10.1155/2014/124564

Marneros, A., \& Akiskal, H. S. (2007). The overlap of affective and schizophrenic spectra. Cambridge, UK: Cambridge University Press.

National Institute for Health and Care Excellence (2009). Schizophrenia: Core interventions in the treatment and management of schizophrenia in adults in primary and secondary care. London, UK: Author.

National Institute for Health and Care Excellence (2014). Psychosis and schizophrenia in adults: Treatment and management. London, UK: Author.

National Social Inclusion Programme (2009). Vision and Progress. Social Inclusion and Mental Health. London, UK: Author.

Padash, Z., Dehnavi, S. R., \& Botlani, S. (2012). The study of efficacy of cognitive therapy based on positive psychology on subjective wellbeing. International Journal of Business and Social Science, 3(10), 202-207.

Pilgrim, D. (1990). Competing histories of madness: Some implications for modern psychiatry. In R. P. Bentall (Ed.), Reconstructing schizophrenia (pp. 211-233). London, UK: Routledge.

Read, J., Seymour, F., \& Mosher, L. (2004). Unhappy families. In J. Read, L. R. Mosher \& R. P. Bentall (Eds.), Models of madness: Psychological, social and biological approaches to schizophrenia (pp. 253-268). Hove, UK: Brunner-Routledge.

Romme, M., \& Escher, S. (1993). Accepting voices. London, UK: Mind Publications.

Royal College of Psychiatrists (2009). Mental health and social inclusion: Position statement. London, UK: Author.

Roth, A., \& Fonagy, P. (2005). What works for whom? A critical overview of psychotherapy research (2nd ed.). New York, NY: The Guilford Press.

Rusk, R. D., \& Waters, L. E. (2013). Tracing the size, reach, impact, and breadth of positive psychology. Journal of Positive Psychology, 8(3), 207-221. http://dx.doi.org/10.1080/17439760.2013.777766

Seligman, M. E. P., \& Csikszentmihalyi, M. (2000). Positive psychology: An introduction. American Psychologist, 55(1), 5-14. http://dx.doi.org/10.1037/0003-066X.55.1.5

Seligman, M. E. P., Rashid, T., \& Parks, A. C. (2006). Positive psychotherapy. American Psychologist, 61(8), 774-788. http://dx.doi.org/10.1037/0003-066X.61.8.774

Siris, S. G. (1991). Diagnosis of secondary depression in schizophrenia: Implications for DSM-IV. Schizophrenia Bulletin, 17(1), 75-98. http://dx.doi.org/10.1093/schbul/17.1.75

Social Exclusion Unit (2004). Mental health and social exclusion. London, UK: Office of the Deputy Prime Minister.

Tew, J., Ramon, S., Slade, M., Bird, V., Melton, J., \& Le Boutillier, C. (2012). Social factors and recovery from mental health difficulties: A review of the evidence. British Journal of Social Work, 3(42), 443-460. http://dx.doi.org/10.1093/bjsw/bcr076

Thompson, S. C. (2002). The role of personal control in adaptive functioning. In C. R. Snyder \& S. J. Lopez (Eds.), Handbook of positive psychology (pp. 202-213). New York, NY: Oxford University Press.

Weiss, N. R., \& Knoster, T. (2008). It may be nonaversive, but is it a positive approach? Relevant questions to ask throughout the process of behavioural assessment and intervention. Journal of Positive Behavior Interventions, 10(1), 72-78. http://dx.doi.org/10.1177/1098300707311389 